\title{
Reducing the rate of psychiatric Re-ADMISsions in Bipolar Disorder using smartphones The RADMIS trial
}

Faurholt-Jepsen, Maria; Tønning, Morten Lindbjerg; Frost, Mads; Martiny, Klaus; Tuxen, Nanna; Rosenberg, Nicole; Busk, Jonas; Winther, Ole; Thaysen-Petersen, Daniel; Aamund, Kate Andreasson Total number of authors:

13

Published in:

Acta Psychiatrica Scandinavica

Link to article, DOI:

10.1111/acps.13274

Publication date:

2021

Document Version

Peer reviewed version

Link back to DTU Orbit

Citation (APA):

Faurholt-Jepsen, M., Tønning, M. L., Frost, M., Martiny, K., Tuxen, N., Rosenberg, N., Busk, J., Winther, O., Thaysen-Petersen, D., Aamund, K. A., Tolderlund, L., Bardram, J. E., \& Vedel Kessing, L. (2021). Reducing the rate of psychiatric Re-ADMISsions in Bipolar Disorder using smartphones The RADMIS trial. Acta Psychiatrica Scandinavica, 143(5), 453-465. https://doi.org/10.1111/acps.13274

\section{General rights}

Copyright and moral rights for the publications made accessible in the public portal are retained by the authors and/or other copyright owners and it is a condition of accessing publications that users recognise and abide by the legal requirements associated with these rights.

- Users may download and print one copy of any publication from the public portal for the purpose of private study or research.

- You may not further distribute the material or use it for any profit-making activity or commercial gain

- You may freely distribute the URL identifying the publication in the public portal 
DR MARIA FAURHOLT-JEPSEN (Orcid ID : 0000-0002-0462-6444)

DR MORTEN LINDBJERG TØNNING (Orcid ID : 0000-0002-0641-682X)

PROFESSOR LARS VEDEL KESSING (Orcid ID : 0000-0001-9377-9436)

Article type : Original Article

Reducing the rate of psychiatric Re-ADMISsions in Bipolar Disorder using smartphones The RADMIS trial

\begin{abstract}
Authors
Maria Faurholt-Jepsen, MD, DMSc ${ }^{1 *}$, Morten Lindbjerg Tønning, MD¹, Mads Frost, PhD², Klaus Martiny, Prof., MD, DMSc ${ }^{1}$, Nanna Tuxen, cand.psych. ${ }^{1}$, Nicole Rosenberg, cand.psych. ${ }^{1}$, Jonas Busk, PhD ${ }^{3}$, Ole Winther, Prof., PhD ${ }^{3,4,5}$, Daniel Thaysen-Petersen, $\mathrm{MD}^{6}$, Kate Andreasson Aamund, MD, PhD , Lizzie Tolderlund7, Jakob Eyvind Bardram, Prof., PhD 2,8 , Lars Vedel Kessing, Prof., MD, DMSc ${ }^{1}$
\end{abstract}

${ }^{1}$ Copenhagen Affective Disorder Research Center (CADIC), Psychiatric Center Copenhagen, Rigshospitalet, Copenhagen, Denmark

${ }_{2}^{2}$ Monsenso Aps, Ny Carlsbergvej 80, Copenhagen, Denmark

${ }^{3}$ Department of Applied Mathematics and Computer Science, Technical University of Denmark, Lyngby, Denmark

${ }^{4}$ Bioinformatics Centre, Department of Biology, University of Copenhagen

${ }^{5}$ Centre for Genomic Medicine, Rigshospitalet, Copenhagen University Hospital

${ }^{6}$ Psychiatric Center Ballerup, Ballerup, Denmark

7 Psychiatric Center North Zealand, Hilleroed, Denmark

${ }^{8}$ Department of Health Technology, Technical University of Denmark, Lyngby, Denmark

*Corresponding author:

Maria Faurholt-Jepsen

Copenhagen Affective Disorder Research Center (CADIC), Psychiatric Center Copenhagen

Rigshospitalet

Blegdamsvej 9

DK- 2100 Copenhagen

Denmark

Telephone: +4538647073

E-mail: maria@faurholt-jepsen.dk

This article has been accepted for publication and undergone full peer review but has not been through the copyediting, typesetting, pagination and proofreading process, which may lead to differences between this version and the Version of Record. Please cite this article as doi: $\underline{10.1111 / \text { acps. } 13274}$

This article is protected by copyright. All rights reserved 
Running title: The effect of smartphone-based monitoring - a RCT

\section{Acknowledgement}

The authors would like to thank the patients for participating in the present trial and the nurses Bente

Støyer, Rikke Møller Jensen and Ida Palmblad Sarauw-Nielsen for running the integrated clinical feedback loop.

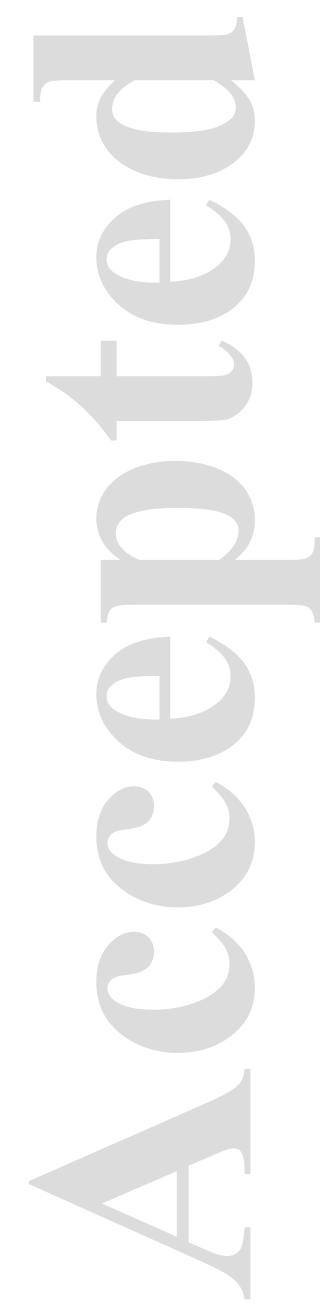

This article is protected by copyright. All rights reserved 


\section{ABSTRACT}

Objectives

The MONARCA I and II trials were negative but suggested that smartphone-based monitoring may increase quality of life and reduce perceived stress in bipolar disorder (BD). The present trial was the first to investigate the effect of smartphone-based monitoring on the rate and duration of readmissions in BD.

\section{Methods}

This was a randomized controlled single-blind parallel-group trial. Patients with BD (ICD-10) discharged from hospitalization in the Mental Health Services, Capital Region of Denmark were randomized 1:1 to daily smartphone-based monitoring including a feedback loop (+ standard treatment) or to standard treatment for 6-months. Primary outcomes: the rate and duration of psychiatric readmissions.

Results

We included 98 patients with BD. In ITT analyses there was no statistically significant difference in rates (hazard rate: $1.05,95 \% \mathrm{Cl}: 0.54 ; 1.91, p=0.88$ ) or duration of readmission between the two groups (B: 3.67, 95\% Cl: $-4.77 ; 12.11, p=0.39$ ). There was no difference in scores on the Hamilton Depression Rating Scale $(B=-0.11,95 \% \mathrm{Cl}:-2.50 ; 2.29, p=0.93)$. The intervention group had higher scores on the Young Mania Rating Scale ( $B: 1.89,95 \% \mathrm{Cl}: 0.0078 ; 3.78, p=0.050)$. The intervention group reported lower levels of perceived stress ( $B:-7.18,95 \% \mathrm{Cl}:-13.50 ;-0.86, p=0.026)$ and lower levels of rumination (B: $-6.09,95 \% \mathrm{Cl}$ :$11.19 ;-1.00, p=0.019)$.

\section{Conclusions}

Smartphone-based monitoring did not reduce rate and duration of readmissions. There was no difference in levels of depressive symptoms. The intervention group had higher levels of manic symptoms, but lower perceived stress and rumination compared with the control group.

Keywords: Bipolar disorder, Randomized controlled trial, Smartphone, Readmission, RADMIS, The Monsenso system.

\section{Significant outcomes}

The effect of daily smartphone-based monitoring and treatment in patients with bipolar disorder for 6 months was investigated in the present randomized controlled single-blind parallel-group trial 
- There were no differences between the two groups in rates or duration of readmission, but the intervention group had higher levels of manic symptoms, reported lower levels of perceived stress and lower levels of rumination

- Due to the limited access to treatment facilities, during recent years, and especially during the COVID-19 pandemic, there has been an rapid increase in the international interest in the use of mHealth technologies within mental health, and smartphone-based monitoring and treatment may present a potential supporting treatment option. However, the evidence has been sparingly investigated.

\section{Limitations}

- Modest sample size

- Only positive significant effect on patient reported outcome measures 


\section{INTRODUCTION}

Bipolar disorder, characterized by recurring affective episodes of depression and (hypo)mania, is one of the most important causes of disability worldwide ${ }^{1}$, and burden society with high health care costs ${ }^{2}$. Patients with bipolar disorder are more frequently hospitalized and readmitted to psychiatric wards than any other patient group ${ }^{3}$. The period following discharge from psychiatric hospitalizations is a high-risk period with a more than 300 times increased risk of suicide and an increased risk of psychiatric readmission ${ }^{4,5}$. Psychopharmacological treatment can reduce the risk of new affective episodes ${ }^{6}$, and this effect may be enhanced by psychological interventions ${ }^{7,8}$. However, studies suggest that due to decreased adherence to psychopharmacological treatment and delayed interventions on prodromal symptoms, the present treatment options does not prevent the progressive course of bipolar disorder ${ }^{4,9}$. Psychological interventions have proven ambient effects on depressive symptoms revealing positive effects in some studies $^{10-12}$, but not in others ${ }^{13-15}$. Specifically targeting depressive rumination, rumination-focused cognitive therapy has shown encouraging results in the treatment of residual depression ${ }^{16}$. However, the number of Cognitive Behavioral Therapy (CBT) therapists is very low compared to the huge demand for CBT. Recently, CBT has been suggested for smartphones ${ }^{17}$, but the effect has never been tested in a randomized controlled randomized trial (RCT) in patients with bipolar disorder.

At an international level within psychiatry there is a gap between the need for treatment and the number of patients receiving treatment. During the last ten years there has been a rapid increase in the interest for mobile health (mhealth (a general term for the use of mobile phones and other wireless technologies in medical care)) technologies within mental health ${ }^{18-22}$. $m$ Health technologies may potentially assist in the gap in need for treatment and available clinicians. Due to the limited access to treatment facilities, during recent years, and especially emphasized during the COVID-19 pandemic and log-down, there has been an rapid increase in the international interest on the potential and importance in advancing the use of various mHealth technologies for both monitoring and treatment within mental health. Smartphones comprise a unique platform for detailed monitoring and treatment on-demand. In relation to bipolar disorder the effect of smartphone-based monitoring has only been investigated in few trials ${ }^{23}$. The MONARCA I trial and the MONARCA II trial, conducted by the authors, were the first clinical trials investigating the effect of smartphone-based monitoring including a clinical feedback loop during six to nine months compared with using a smartphone for usual communicative purposes in patients with bipolar disorder ${ }^{20,24}$. The clinical feedback loop comprised study nurses, who examined the patients' smartphone data on a regular basis using a secure webpage. The patients were contacted by the study nurses based on the patients' individual needs and in case of signs of deterioration. The study nurses then gave advice based on the patients' current clinical state. Overall, in the MONARCA I and the MONARCA II trial there was no effect of 
smartphone-based monitoring on the severity of depressive and manic symptoms. Sub-analyses on the primary outcome measures suggested that smartphone-based monitoring might sustain depressive symptoms and episodes, but on the other hand reduce manic symptoms and episodes. On the other hand, results also suggested that smartphone-based monitoring resulted in higher quality of life and lower perceived stress ${ }^{24}$.

Depressive and manic episodes are associated with observable alterations in several behavioural components (e.g. activity level and level of social communication). Some of these behavioural components may be reflected in automatically generated objective smartphone data (such as phone usage, social activity, and mobility), which we previous have shown are associated with the level of clinically evaluated depressive and manic symptoms, are able to discriminate between affective states (state marker) and may represent a potential diagnostic marker in comparison with healthy control individuals (trait marker) ${ }^{25-27}$.

\section{Hypotheses}

Following the MONARCA I trial and the MONARCA I| trial, we adjusted the smartphone-based monitoring system. A new integrated clinical feedback loop, based on real-time prediction models including both subjective measures and automatically generated objective smartphone data, as well as inclusion of CBT modules was established and implemented in the new version of the smartphone-based system (now called the Monsenso system). We conducted the Re-ADMISsions (RADMIS) RCT and hypothesized, that daily smartphone-based monitoring and mood prediction including the new integrated clinical feedback loop and CBT modules would reduce the rate and duration of psychiatric readmissions more than standard treatment in patients with bipolar disorder discharged from psychiatric hospitalization.

\section{Aim of the trial}

The objective of the present trial was to investigate in a randomized controlled single-blind parallel-group trial whether the use of the Monsenso system, would reduce the rate and duration of psychiatric readmissions more than standard treatment in adult patients with bipolar disorder (primary outcomes). This was the RADMIS trial. Secondary, we aimed to investigate if the Monsenso system would reduce the severity of clinically rated depressive and manic symptoms and the number of affective episodes, improve psychosocial functioning, quality of life, severity of self-assessed affective symptoms, recovery, empowerment, adherence to medication, well-being and satisfaction with care, and reduce perceived stress, rumination, and worrying more than standard treatment without a smartphone-based system in adult patients with bipolar disorder. 


\section{MATERIAL AND METHODS}

The RADMIS trial is reported according to the CONsolidated Standards Of Reporting Trials (CONSORT) statement and Standard Protocol Items: Recommendations for Interventional Trials (SPIRIT) ${ }^{28}$. Details concerning the design and methods of the trial can be found elsewhere ${ }^{29}$.

\section{Settings, population and design}

All patients with a diagnosis of bipolar disorder who were hospitalized due to an affective episode and being discharged from psychiatric hospitalization from one of five psychiatric centres at the Mental Health Services, Capital Region of Denmark, Denmark (Psychiatric Center Copenhagen; Psychiatric Center Glostrup; Psychiatric Center Amager; Psychiatric Center Ballerup; Psychiatric Center North Zealand) in the period from May 2017 to August 2019 were invited to participate in the trial. Inclusion criteria: Age above 18 years; bipolar disorder diagnoses according to ICD-10 using Schedules for Clinical assessments in Neuropsychiatry (SCAN) 30 ; discharged from a psychiatric hospital in The Capital Region of Denmark following an affective episode (depression, mania or mixed episode).

Exclusion criteria: Pregnancy and a lack of Danish language skills. The RADMIS trial had a randomized controlled single-blind parallel-group design with a balanced allocation ratio of adult patients with bipolar disorder.

\section{Allocation, randomization and blinding}

A list of random allocation numbers generated by Pharma Consulting Group (http://www. pharmaconsultinggroup.com) with random block sizes between 6-10 was used. The allocation sequence was concealed from the researchers (MFJ and MLT) and from the study nurses responsible for the integrated feedback loop. In addition to standard treatment patients were randomized with a balanced allocation of 1:1 to either 1 ) daily use of the Monsenso system including the integrated feedback loop based on a combination of subjective self-monitored measures and automatically generated objective smartphone data and CBT modules (the intervention group) or to 2) normal use of smartphones (the control group) during a six months follow-up period. Randomization was stratified according to the discharging Psychiatric Center and the number of previous psychiatric hospitalizations ( $\leq 3$ or $>3$ ). The flow diagram of the RADMIS trial is presented in Figure 1. No changes in were made after trial commencement.

Owing to the type of intervention in the RADMIS trial, the patients, the patients' health care provider and the study nurses were aware of the allocated randomization group. Thus, the trial was single-blinded. The 
allocation status was blinded to the researchers (MFJ, MLT) conducting outcome assessments, statistical analyses, interpretation of results and writing of the paper.

\section{Intervention}

The intervention group (further details in supplementary data and ${ }^{29}$ )

At baseline, the Monsenso system was installed on the patients' own or a smartphone borrowed by the trial. The patients were prompted by an alarm in system to conduct daily self-monitoring using the Monsenso system. The symptoms, which the patients had to evaluated on a daily basis, included mood, level of activity, duration of sleep, medication intake etc. (Figure 2). Automatically generated objective smartphone data including data on phone usage, social activity and mobility were collected and included in the clinical feedback loop. The CBT modules included psychoeducation, cognitive restructuring, and rumination-focused CBT.

The integrated clinical feedback loop was comprised by the study nurses, who regularly examined the collected smartphone data on a webpage. Based on the patients' individual needs and collected data the study nurses contacted the patients and gave advice based on the patients' current clinical state (supplementary data).

\section{INSERT FIGURE 2 HERE}

Figure 2. The Monsenso system for smartphones

The control group

The patients in the control group used their own smartphone or a smartphone borrowed by the trial for usual communicative activities.

All patients continued with their standard treatment. Outpatient treatment in Denmark is given either by the general practitioner, private psychiatrist, or in a multidisciplinary outpatient clinic with psychiatrists, psychologists, and nurses.

\section{Outcome measures}

All included outcomes were defined a priori ${ }^{29}$. The primary outcomes were defined as differences between the intervention group and the control group in rate and duration of psychiatric readmissions collected using the patients' electronic health record. The secondary outcomes were defined as differences between the two groups in the severity of clinically evaluated depressive and manic symptoms 
assessed using the Hamilton Depression Rating scale 17-items (HDRS) and the Young Mania Rating Scale (YMRS), respectively ${ }^{31,32}$, psychosocial functioning according to the Functional Assessment Short Test $(\text { FAST })^{33}$, and the number of depressive and manic episodes defined as HDRS $\geq 13$ and $Y M R S \geq 13$, respectively.

The tertiary outcomes were differences between the two groups in perceived stress according to Cohen's Perceived Stress Scale (PSS) ${ }^{34}$, quality of life according to the WHO Quality of Life-BREF (WHOQOL-BREF) ${ }^{35}$, patient-reported depressive symptoms according to Becks Depressive Inventory (BDI) ${ }^{36}$, patient-reported depressive symptoms according to the Hamilton Depression Self-rating Scale 6-item (HDRS-6) ${ }^{37}$, patientreported manic symptoms according to the Altman Self Rating scale for Mania (ASRM) ${ }^{38}$, recovery according

to the Recovery Assessment Scale ${ }^{39}$, empowerment according to Rogers empowerment scale ${ }^{40}$, adherence to medication according to the Medicine Adherence Rating Scale ${ }^{41}$, well-being according to the WHO (five) well-being index ${ }^{42}$, rumination according to the Ruminative Response Scale (RRS) ${ }^{40}$, worrying according to the Penn State Worry Questionnaire (PSWQ) ${ }^{43}$, behavioral activation according to the Behavioral Activation Scale (BAS) ${ }^{44}$ and satisfaction with care according to the Verona Satisfaction Scale-Affective Disorder (VSS$A)^{45}$. Based on findings from our previous trials (the MONARCA I trial and the MONARCA || trial) ${ }^{20,24}$, we conducted sub-analyses investigating differences between the intervention group and the control group in patients presenting with depressive or manic symptoms at baseline (defined as HDRS $\geq 7$ or YMRS $\geq 7$ ).

At baseline, after 3 months and 6 months clinical evaluation of the included patients were carried out by Medical Doctor researchers (MFJ and MLT) who were not involved in the treatment of the patients, and who at all times were kept blinded to the patients' allocation status.

\section{Statistical methods}

A priori the statistical analyses were defined ${ }^{29}$, and conducted using an Intention-to-treat (ITT) approach. Analyses of the hazard rate of readmission (primary outcome) were investigated in a Cox regression model with reasons for censoring being death, leaving the country, or end of study. Event was defined as the first day of readmission. Rate of readmission over the 6 months trial period was illustrated in a Kaplan-Meier plot (Figure 3 ) and difference between the intervention group and the control group was tested in a logrank test. Time was calculated from the day of discharge from psychiatric hospitalization. Proportional hazards were assessed by goodness of fit models and graphical presentations. 
Secondary and tertiary outcomes: All continuous outcome measures were analyzed in a two-level linear mixed effect model allowing for both intra-individual and inter-individual variation of the outcome variables. Level one represented repeated measures of symptoms (e.g. HDRS, YMRS) and level two represented inter-individual variation. The model included a fixed effect of visit number and a patientsspecific random effect (Table 2 \& 3). First, we conducted unadjusted models (but adjusted for differences in baseline values of the outcome variables and the two stratification variables. Second, we conducted models adjusted for age and sex. Finally, we conducted models further adjusting for scores on the HDRS and the YMRS (except for measures of depression and mania).

Differences in rates of relapse of episodes during the study were investigated using Cox regression analysis calculating the Hazard Rate (HR) with reasons for censoring being death or date of drop out (Table 2). Model assumptions with analyses of residuals and covariance were calculated and assessed for all outcomes. Missing items in the questionnaires were not included in the summed scores, and no imputations or assumptions on missing items were made. Single questionnaires with more than $20 \%$ missing items were discarded. Statistical interactions between randomization group and visit number on outcome variables were investigated and reported if present. The overall hypotheses of the study included several outcome measures, and thus the present study should be considered as hypotheses generating. The results need further replication. Consequently, we did not account for multiple testing in the statistical models. The threshold for statistical significance was $p \leq 0.05$ (two-tailed). STATA version 13 (StataCorp LP, College Station, TX, USA) was used for all analyses.

\section{Ethical considerations}

The RADMIS trial was approved by the Regional Ethics Committee in The Capital Region of Denmark and the data agency, Capital Region of Copenhagen (H-16046093, RHP-2017-005, I-Suite:05365) and registered at ClinicalTrials.gov (NCT03033420). The law on handling of personal data was respected. All data collected by researchers was stored in the Research Electronic Data Capture (REDCap) system ${ }^{46,47}$. Data from smartphones was stored by Monsenso subject to a data management agreement between Monsenso and The Capital Region of Denmark. The patients did not receive any economic compensation for participating. The trial complied with the Declaration of Helsinki of 1975, as revised in 2008. 


\section{RESULTS}

A total of 382 patients discharged from psychiatric hospitalization in the Capital Region of Denmark from May 2017 to August 2019 were assessed for eligibility. Of these, a total of 284 patients were not included since they a) declined to participate due to not wanting to participate in a research study/not wanting an additional clinical outpatient contact or b) it was not possible to establish contact with the patient. The last patients' last visit was in February 2020. A total of 98 patients were randomized 1:1 to either the intervention group (active use of the Monsenso system) or to control group (use of a smartphone for usual communication) in addition to standard treatment for six months and left for ITT analyses. Of these, 11 dropped out during the trial (intervention group: 6; control group: 5) due to no specific reasons, apart from one patient who withdraw due to change in smartphone type during the study. A total of 34 patients had a discontinued visit at follow-up with the researchers (intervention group: 10; control group: 24). Details concerning the flow of patients in the RADMIS trial are presented in Figure 1. The included patients had a mean age of $42.69(S D=13.46)$ years and $59.18 \%(n=58)$ were women. Further details concerning clinical and socio-demographic characteristics are presented in Table 1.

The patients in the intervention group had an average adherence to daily smartphone-based selfmonitoring $80.6 \%$ of the days during the six-month trial period. During the trial period the patients in the intervention group registered a mean mood of -0.30 (SD 0.65). The patients in the intervention group had regular contact with study nurses and registrations throughout the trial period but with variation in use depending on the patients' needs and abilities. The main communication form between the patients and the study nurses was via phone calls and text messages.

\section{Rate and duration of psychiatric readmissions - primary outcomes}

A total of 36 patients were readmitted during the trial $(n=17$ in the intervention group and $n=19$ in the control group). A total of 25 patients were readmitted once $(n=12$ in the intervention group and $n=13$ in the control group), 10 patients were readmitted twice ( $n=5$ in the intervention group and $n=5$ in the control group), and one patient was readmitted three times (in the control group). Among the patients who were readmitted, the mean duration of readmission was 11.33 (SD 21.73) days (intervention group mean=11.68 $(S D=25.59)$ days and control group mean=9.06 ( $S D=17.62)$ days). Differences in rates of psychiatric readmissions between the intervention group and the control are presented in Table 2 and Figure 3 . The Hazard Rate for readmission in the intervention group compared with the control group was 1.05 (95\% Cl: $0.54 ; 1.91, p=0.88)$. The mean survival time calculated as the area under the Kaplan-Meier curve was 
166.32 (95\% Cl: $145.41 ; 187.23)$ days in the intervention group and 170.46 (95\% Cl: $151.52 ; 189.41)$ days in the control group. The mean duration of days to readmission was 75.12 (SD=43.28) days in the intervention group and 89.11 (SD=48.29) days in the control group. There was no statistically significant difference in rate of psychiatric readmission between the two groups (log Rank test, $p=0.99$ ). None of the patients were excluded from the analyses due to censoring apart from end of study. Differences in accumulated duration of psychiatric readmissions are presented in Table 2. There were no statistically significant differences in accumulated duration of psychiatric readmissions between the intervention group and the control group in both the unadjusted and adjusted model (adjusted model: $B=3.67,95 \% \mathrm{Cl}:-4.77 ; 12.11, p=0.39$ ).

In exploratory analyses stratifying according to sex and age $>$ or $<25$ years or bipolar disorder subtype I or II, there were no statistically significant differences in any of the primary outcomes between the intervention group and the control group.

\section{---- INSERT FIGURE 3 HERE}

Figure 3. Kaplan-Meier curve of the ITT analysis on time to psychiatric readmission

\section{Secondary and tertiary outcomes}

Analyses on secondary and tertiary outcomes are presented in Table 2 and Table 3. There were no statistically significant differences between the intervention group and the control group in scores on the HDRS (adjusted model: $B=-0.11,95 \% \mathrm{Cl}:-2.50 ; 2.29, p=0.93$ ). Nevertheless, in a subgroup analysis of patients with an HDRS score $\geq 7$ at baseline there was a statistically significantly higher level of depressive symptoms according to the HDRS in the intervention group compared with the control group (adjusted model: $B=2.55,95 \% \mathrm{Cl}: 0.08 ; 5.02, p=0.05)$. Further, there was a statistically significantly higher level of manic symptoms according to the YMRS in the intervention group compared with the control group (adjusted model: $B=1.89,95 \% \mathrm{Cl}: 0.0078 ; 3.78, p=0.05)$. In a subgroup analysis of patients with a YMRS score $\geq 7$ at baseline there was a statistically significantly higher level of manic symptoms according to the YMRS in the intervention group compared with the control group (adjusted model: $B=4.17,95 \% \mathrm{Cl}$ : 1.07; $7.27, p=0.008)$. In patients not in a current affective episode at baseline, there were no differences between the intervention group and the control group in rates of depressive episodes, manic episodes and episodes regardless polarity during the trial period (all $p$ values> 0.24 ).

There was no statistically significant difference between the intervention group and the control group in functioning according to the FAST (adjusted model: $B=-1.91,95 \% \mathrm{Cl}:-5.41 ; 1.59, p=0.29$ ). 
Regarding tertiary outcome measures, there was a statistically significantly lower level of rumination according to the Ruminative Response Scale (adjusted model: $B=-6.09,95 \% \mathrm{Cl}:-11.19 ;-1.00, p=0.019$ ), lower perceived stress according to scores on the Cohen's Perceived stress scale (adjusted model: $B=-7.18$, 95\% Cl: $-13.50 ;-0.86, p=0.026)$ and lower self-rated manic symptoms according to the Altman Self-rating Scale for Mania (adjusted model: $B=-1.43,95 \% \mathrm{Cl}:-2.77 ;-0.099, p=0.031$ ) in the intervention group compared with the control group. There were no statistically significant differences in any of the other tertiary outcomes concerning quality of life, self-rated depressive symptoms, recovery, empowerment, adherence to medication, well-being, worrying, behavioral activation and satisfaction with care (all pvalues>0.06).

Analyses on interactions between randomization group and time (visit number) on the outcome variables were statistically non-significant (all p values> 0.11 ), and therefore not reported.

In exploratory analyses stratifying according to sex and age $>$ or $<25$ years or bipolar disorder subtype I or II, there were no statistically significant differences in any of the secondary and tertiary outcomes between the intervention group and the control group. 


\section{DISCUSSION}

Findings from the present trial

This RCT investigated the effect of daily smartphone-based monitoring on the rate and duration of psychiatric readmissions in patients with bipolar disorder discharged from psychiatric admissions. During a six-month period, in this single-blinded RCT patients were randomized to either a daily smartphone-based monitoring system including a clinical feedback loop integrating subjective as well as automatically generated objective smartphone data and CBT modules in addition to standard treatment, or to standard treatment alone. Overall, we found no effect on rate and duration of psychiatric readmissions. Further, we found no effect on the severity of depressive symptoms according to scores on the HDRS. Surprisingly, compared with the control group patients in the intervention group presented with statistically significantly higher levels of manic symptoms according to the YMRS. Furthermore, in a subgroup analysis of patients with a HDRS score $\geq 7$ at baseline, patients in the intervention group presented with higher level of depressive symptoms according to the HDRS. In contrast, we found that patients in the intervention group reported statistically significantly lower levels of perceived stress and lower levels of rumination compared with the control group.

\section{Findings from the present trial in context}

Due to the limited access to treatment facilities, during the past ten years, and especially during the COVID19 pandemic, there has been an increase in the international interest in the use of mHealth technologies within mental health ${ }^{19,48,49}$. Despite the hype, there has been conducted very few trial investigating the effect of smartphone-based monitoring and treatment ${ }^{23}$. Few previous studies have investigated the effect of smartphone-based monitoring compared with paper-based monitoring ${ }^{50}$, or investigated the effect of smartphone-based treatment compared with clinic-based group intervention ${ }^{51,52}$. Notably, these trials did not investigate the effect of daily smartphone-based monitoring including a clinical feedback loop compared with standard treatment, the trials did not include a clinically well-characterized population or did not report on clinician evaluated outcome measures. Currently, a few trials investigating the effect of smartphone-based psychoeducation ${ }^{53}$ and smartphone-based automated feedback on ambulatory assessed early warning signs ${ }^{54}$ are ongoing. The authors of the present trial have previously conducted the MONARCA I trial and the MONARCA II trial investigating the effect of daily smartphone-based monitoring and treatment including a clinical feedback loop in bipolar disorder ${ }^{20,24}$. Findings from these trials were negative with no effect on clinically evaluated symptoms (both depressive and manic). However, analyses of secondary outcome measures and sub-analyses suggested that smartphone-based monitoring and treatment might reduce manic symptoms and reduce the risk of manic episodes but on the other hand 
sustain depressive symptoms and increase the risk of depressive episodes. Furthermore, findings

suggested, that smartphone-based monitoring and treatment may result in higher quality of life and lower perceived stress.

Interestingly, some of the findings from the present RADMIS trial in relation to the secondary outcome measures are in line with findings from the MONARCA I trial ${ }^{24}$ and the MONARCA II trial ${ }^{20}$. Thus, the finding that patients in the intervention group presenting with depressive symptoms at baseline presented with higher levels of depressive symptoms during follow-up are in line with findings from the MONARCA I trial and the MONARCA II trial. Due to negative processing bias induced by the daily confrontation with the depressive symptoms it could be that continuous daily self-monitoring of ongoing depressive symptoms may sustain depressive symptoms and, in turn, may have induced or increased fear of not recovering. Thus, continuous monitoring of depressive symptoms might have a maintenance effect on symptoms themselves. Patients in the prodromal phase of a depressive episode may experience depressive ruminations with potential further risk of worsening and escalation of depressive symptoms. Further to this point, treating depressive prodromes in bipolar disorder is a difficult process and few drugs have proved effects in bipolar depression ${ }^{14,55}$.

The finding from the present trial that patients in the intervention group presented with higher levels of manic symptoms according to scores on the YMRS (although only nearly borderline significant (Table 2)) contrasts with the finding of lower patient-reported manic symptoms according to the ASRM and with previous findings $\mathrm{s}^{20,24}$. However, these findings are based on small number of patients and conduction of statistical sub-analyses, and thus should be interpreted with caution. Further, some findings that are not in concordance with previous findings may be noise. The findings could be due to the low number patients in each allocation group of the trial. The finding that patients in the intervention group presented with lower levels of patient-reported perceived stress is in line with findings from the MONARCA II trial. The finding from the present RADMIS trial, that smartphone-based monitoring and treatment may reduce the level of rumination is interesting. A recent meta-analysis found rumination, reflecting repetitive thoughts about emotionally relevant experiences, to be a significant process in bipolar disorder ${ }^{56}$, highlighting the importance of interventions to reduce rumination in bipolar disorder. Furthermore, concerning the treatment of residual depression, rumination focused cognitive therapy and concreteness training (a facilitated self-help intervention for increasing specificity of processing in patients with depression) have shown encouraging results ${ }^{16}$.

\section{Limitations}


The overall findings from the present RADMIS trial are negative. Despite the hype concerning mHealth interventions, we did not find an effect of daily smartphone-based treatment for patient with bipolar disorder. It could be that there would be an effect of smartphone-based treatment including an integrated feedback loop on other outcome measures, which we did not include in the present trial. During recent years there has been an increasing interest in Patient-Reported Outcome Measures (PROMS) as valid indicators of effect of treatment interventions in mental health research ${ }^{57,58}$. The findings from the present trial on reduced patient-reported rumination, perceived stress and lower patient-reported manic symptoms could indicate relevant improvement in patient-reported quality of care, which is not reflected in clinicians assessed outcome measure on severity of symptoms and readmission. However, these finding were based on tertiary outcome measures. Furthermore, the patients included in the present trial had a rather high number of previous affective episodes. Previous studies have suggested that psychotherapies may be more effective preventing relapse in patients with fewer previous affective episodes, and thus it may be that including patient with fewer previous episodes would have resulted in other findings concerning the primary outcomes measures ${ }^{59}$. In addition, it has been suggested that mHealth technologies may have potentially limited effectiveness on "hard" outcome measures such as relapse or readmission in patients where medication or face-to-face psychotherapy have already failed to prove useful for these "hard" outcome measures ${ }^{60}$. The patients included in the present trial were unstable and included at discharged following hospitalization for an affective episode. We did not include information on whether some of the patients had previously received formalized psychoeducation, which may affect the effect of the smartphone-based psychoeducation module. However, a previous trial by the authors including patients with bipolar disorder who had received formalized psychoeducation did not find an effect of smartphone-based treatment on the severity of depressive and manic symptoms ${ }^{20}$. Due to difficulties in recruitment, the present trial did not include the intended sample size of 200 patients despite prolonging the recruitment period. Nevertheless, post hoc statistical analyses on the power of the trial showed a sufficient power to exclude a type II error at a p-value of 0.05 with a sample size of 98 patients. There may be an effect of different components of the smartphone-based system used in the present trial. However, from the present trial it is not possible to address the potential effect of different components of system. During the RADMIS trial, minor adjustments were made to the smartphone-based monitoring system. However, these minor adjustments are found to reflect real-life settings. The included patients were randomized stratified according to the number of previous psychiatric hospitalizations ( $\leq 3$ or $>3$ ) to prevent imbalance between treatment groups for factors which could potentially influence effect of treatment. However, the exact cut-off was arbitrary, and it cannot be excluded that another cut-off would 
have resulted in other findings. A rather large number of patients were assessed for eligibility but not included (flow diagram). This may have affected the generalizability of the findings.

\section{Advantages}

The present trial used a smartphone-based system developed and tested by our group in multiple studies $25-27,61-63$, which has been shown usable and useful by patients with bipolar disorder with a high selfassessment adherence $(73-97 \%)^{64}$. Further, we used a single-blind design, and thus the outcome measures collected by the researchers were blinded to allocation. The trial was pragmatic with few exclusion criteria resembling patients with bipolar disorder in general. The findings from the present trial are found to reflect smartphone-based monitoring during real-world settings.

\section{Conclusion}

In patients discharged from psychiatric hospitalization due to an affective episode as part of bipolar disorder, daily smartphone-based monitoring and treatment, including a clinical feedback loop, did not reduce the rate and duration of psychiatric readmission. Analyses on secondary and tertiary outcome measures showed no difference in depressive symptoms overall, but higher levels of manic symptoms, higher level of depressive symptoms in sub-analyses, and lower perceived stress and rumination in the intervention group compared with the control group. Findings were based on small numbers and should be interpreted with caution. Potential negative effect should be investigated further in future trials.

\section{Conflicts of interest}

MFJ, MLT, KM, NT, NR, JB, OW, DTP, KAA, and LT have no competing interests. MF and JEB are co-founders and shareholders in Monsenso. LVK has been a consultant for Lundbeck within the past three years.

\section{Authors' contributions}

LVK and JEB obtained funding for the trial and conceived the trial together with MFJ. MFJ, MLT and LVK conducted the trial, analysed data and authored the first draft of the manuscript. All authors contributed to and approved the final version of the manuscript.

\section{Funding}

The RADMIS trial was funding by Innovation Fund Denmark (5164-00001B9). The funding organization had no role in the design and conduct of the study; collection, management, analysis, and interpretation of the data; preparation, review, or approval of the manuscript; and decision to submit the manuscript for publication. 


\section{REFERENCES}

15. Scott, J. \& Colom, F. Gaps and limitations of psychological interventions for bipolar disorders. Psychother. Psychosom. 77, (2008).

16. Watkins, E. R. Depressive Rumination: Investigating Mechanisms to Improve Cognitive Behavioural Treatments. Cogn. Behav. Ther. 38, 8-14 (2009).

17. Ly, K. H. et al. Experiences of a guided smartphone-based behavioral activation therapy for depression: A qualitative study. Internet Interv. 2, 60-68 (2015).

18. Faurholt-Jepsen, M., Munkholm, K., Frost, M., Bardram, J. E. \& Kessing, L. V. Electronic self-monitoring of mood using IT platforms in adult patients with bipolar disorder: A systematic review of the validity and evidence. BMC Psychiatry 16, 7 (2016).

19. Wang, K., Varma, D. S. \& Prosperi, M. A systematic review of the effectiveness of mobile apps for monitoring and management of mental health symptoms or disorders. J. Psychiatr. Res. 107, 73-78 (2018).

20. Faurholt-Jepsen, M. et al. The effect of smartphone-based monitoring on illness activity in bipolar disorder: the MONARCA II randomized controlled single-blinded trial. Psychol. Med. 1-11 (2019) doi:10.1017/\$0033291719000710.

21. Bauer, M. et al. Smartphones in mental health: a critical review of background issues, current status and future concerns. Int. J. Bipolar Disord. 8, 2 (2020).

22. Bardram, J. E. \& Matic, A. A Decade of Ubiquitous Computing Research in Mental Health. IEEE Pervasive Comput. 19, 62-72 (2020).

23. Tønning, M. L., Kessing, L. V., Bardram, J. E. \& Faurholt-Jepsen, M. Methodological Challenges in Randomized Controlled Trials on Smartphone-Based Treatment in Psychiatry: Systematic Review. J. Med. Internet Res. 21, e15362 (2019).

24. Faurholt-Jepsen, M. et al. Daily electronic self-monitoring in bipolar disorder using smartphones - the MONARCA I trial: a randomized, placebo-controlled, single-blind, parallel group trial. Psychol. Med. 45, 2691-2704 (2015). 
25. Faurholt-Jepsen, M. et al. Behavioral activities collected through smartphones and the association with illness activity in bipolar disorder. Int. J. Methods Psychiatr. Res. 25, 309-323 (2016).

Faurholt-Jepsen, M. et al. Smartphone data as an electronic biomarker of illness activity in bipolar disorder. Bipolar Disord. Nov.; 17, 715-28 (2015).

27. Faurholt-Jepsen, M. et al. Objective smartphone data as a potential diagnostic marker of bipolar disorder. Aust. N. Z. J. Psychiatry 0004867418808900 (2018) doi:10.1177/0004867418808900.

28. Moher, D. et al. CONSORT 2010 explanation and elaboration: updated guidelines for reporting parallel group randomised trials. BMJ 340 , c869 (2010).

Faurholt-Jepsen, M. et al. Reducing the rate and duration of Re-ADMISsions among patients with unipolar disorder and bipolar disorder using smartphone-based monitoring and treatment - the RADMIS trials: study protocol for two randomized controlled trials. Trials 18, 277 (2017). Wing, J. K. et al. SCAN. Schedules for Clinical Assessment in Neuropsychiatry. Arch. Gen. Psychiatry 47, $589-593$ (1990). Hamilton, M. Development of a rating scale for primary depressive illness. Br. J. Soc. Clin. Psychol. 6, 278-296 (1967).

32. Young, R. C., Biggs, J. T., Ziegler, V. E. \& Meyer, D. A. A rating scale for mania: reliability, validity and sensitivity. Br. J. Psychiatry 133, 429-435 (1978). Rosa, A. R. et al. Validity and reliability of the Functioning Assessment Short Test (FAST) in bipolar disorder. Clin. Pract. Epidemiol. Ment. Health CP EMH 3, 5 (2007). Cohen, S., Kamarck, T. \& Mermelstein, R. A global measure of perceived stress. J. Health Soc. Behav. 24, 385-396 (1983). WHO. Development of the World Health Organization WHOQOL-BREF quality of life assessment. The WHOQOL Group. Psychol. Med. 28, 551558 (1998). BECK, A. T., WARD, C. H., MENDELSON, M., MOCK, J. \& ERBAUGH, J. An inventory for measuring depression. Arch. Gen. Psychiatry 4, 561-571 (1961).

37. Bech, P., Gefke, M., Lunde, M., Lauritzen, L. \& Martiny, K. The Pharmacopsychometric Triangle to Illustrate the Effectiveness of T-PEMF Concomitant with Antidepressants in Treatment Resistant Patients: A Double-Blind, Randomised, Sham-Controlled Trial Revisited with Focus on the Patient-Reported Outcomes. Depress. Res. Treat. 2011, (2011).

38. Young, R. C., Biggs, J. T., Ziegler, V. E. \& Meyer, D. A. A rating scale for mania: reliability, validity and sensitivity. Br. J. Psychiatry 133, 429-435 (1978).

39. Corrigan, P. W., Salzer, M., Ralph, R. O., Sangster, Y. \& Keck, L. Examining the factor structure of the recovery assessment scale. Schizophr. Bull. 30, 1035-1041 (2004).

40. Rogers, E. S., Chamberlin, J., Ellison, M. L. \& Crean, T. A consumer-constructed scale to measure empowerment among users of mental health services. Psychiatr. Serv. Wash. DC 48, 1042-1047 (1997).

41. Thompson, K., Kulkarni, J. \& Sergejew, A. A. Reliability and validity of a new Medication Adherence Rating Scale (MARS) for the psychoses. Schizophr. Res. 42, 241-247 (2000).

42. Bech, P., Olsen, L. R., Kjoller, M. \& Rasmussen, N. K. Measuring well-being rather than the absence of distress symptoms: a comparison of the SF-36 Mental Health subscale and the WHO-Five well-being scale. Int. J. Methods Psychiatr. Res. 12, 85-91 (2003).

43. Meyer, T. J., Miller, M. L., Metzger, R. L. \& Borkovec, T. D. Development and validation of the penn state worry questionnaire. Behav. Res. Ther. 28, 487-495 (1990).

44. Raes, F., Hoes, D., Van Gucht, D., Kanter, J. W. \& Hermans, D. The Dutch version of the Behavioral Activation for Depression Scale (BADS): psychometric properties and factor structure. J. Behav. Ther. Exp. Psychiatry 41, 246-250 (2010).

45. Kessing, L. V., Hansen, H. V., Ruggeri, M. \& Bech, P. Satisfaction with treatment among patients with depressive and bipolar disorders. Soc. Psychiatry Psychiatr. Epidemiol. 41, 148-155 (2006). Harris, P. A. et al. The REDCap consortium: Building an international community of software platform partners. J. Biomed. Inform. 95, 103208 (2019).

47. Harris, P. A. et al. Research electronic data capture (REDCap)--a metadata-driven methodology and workflow process for providing translational research informatics support. J. Biomed. Inform. 42, 377-381 (2009).

48. Anthes, E. Mental health: There's an app for that. Nature 532, 20-23 (2016). 
Insel, T. R. Digital Phenotyping: Technology for a New Science of Behavior. JAMA 318, 1215-1216 (2017).

Depp, C. A. et al. Augmenting psychoeducation with a mobile intervention for bipolar disorder: A randomized controlled trial. J. Affect. Disord. 174, 23-30 (2015).

51. Ben-Zeev, D. et al. Mobile Health (mHealth) Versus Clinic-Based Group Intervention for People With Serious Mental Illness: A Randomized Controlled Trial. Psychiatr. Serv. Wash. DC appips201800063 (2018) doi:10.1176/appi.ps.201800063.

Ben-Zeev, D. et al. Transdiagnostic Mobile Health: Smartphone Intervention Reduces Depressive Symptoms in People With Mood and Psychotic Disorders. JMIR Ment. Health 6, e13202 (2019).

53. Hidalgo-Mazzei, D. et al. Self-monitoring and psychoeducation in bipolar patients with a smart-phone application (SIMPLe) project: design, development and studies protocols. BMC Psychiatry 15, (2015).

Mühlbauer, E. et al. Effectiveness of smartphone-based ambulatory assessment (SBAA-BD) including a predicting system for upcoming episodes in the long-term treatment of patients with bipolar disorders: study protocol for a randomized controlled single-blind trial. $B M C$ Psychiatry 18, 349 (2018).

55. Bahji, A., Ermacora, D., Stephenson, C., Hawken, E. R. \& Vazquez, G. Comparative efficacy and tolerability of pharmacological treatments for the treatment of acute bipolar depression: A systematic review and network meta-analysis. J. Affect. Disord. 269, 154-184 (2020). Kovács, L. N. et al. Rumination in major depressive and bipolar disorder - a meta-analysis. J. Affect. Disord. 276, 1131-1141 (2020). Roe, D., Mazor, Y. \& Gelkopf, M. Patient-reported outcome measurements (PROMs) and provider assessment in mental health: a systematic review of the context of implementation. Int. J. Qual. Health Care J. Int. Soc. Qual. Health Care (2019) doi:10.1093/intqhc/mzz084. Kynoch, K., Ramis, M.-A. \& Khalil, H. PREMS and PROMS data within the acute health care context: a scoping review protocol. JBI Evid. Synth. (2020) doi:10.11124/JBISRIR-D-19-00355.

59. Scott, J., Colom, F. \& Vieta, E. A meta-analysis of relapse rates with adjunctive psychological therapies compared to usual psychiatric treatment for bipolar disorders. Int. J. Neuropsychopharmacol. Off. Sci. J. Coll. Int. Neuropsychopharmacol. CINP 10, 123-129 (2007). Hidalgo-Mazzei, D., Llach, C. \& Vieta, E. mHealth in affective disorders: hype or hope? A focused narrative review. Int. Clin. Psychopharmacol. 35, 61-68 (2020).

61. Faurholt-Jepsen, M. et al. Voice analysis as an objective state marker in bipolar disorder. Transl. Psychiatry 6, e856 (2016).

62. Stanislaus, S. et al. Mood instability in patients with newly diagnosed bipolar disorder, unaffected relatives, and healthy control individuals measured daily using smartphones. J. Affect. Disord. 271, 336-344 (2020).

63. Melbye, S. A. et al. Mood, activity, and sleep measured via daily smartphone-based self-monitoring in young patients with newly diagnosed bipolar disorder, their unaffected relatives and healthy control individuals. Eur. Child Adolesc. Psychiatry (2020) doi:10.1007/s00787-02001611-7.

64. Faurholt-Jepsen, M. et al. Smartphone-based self-monitoring in bipolar disorder: evaluation of usability and feasibility of two systems. Int. J. Bipolar Disord. 7, 1 (2019).
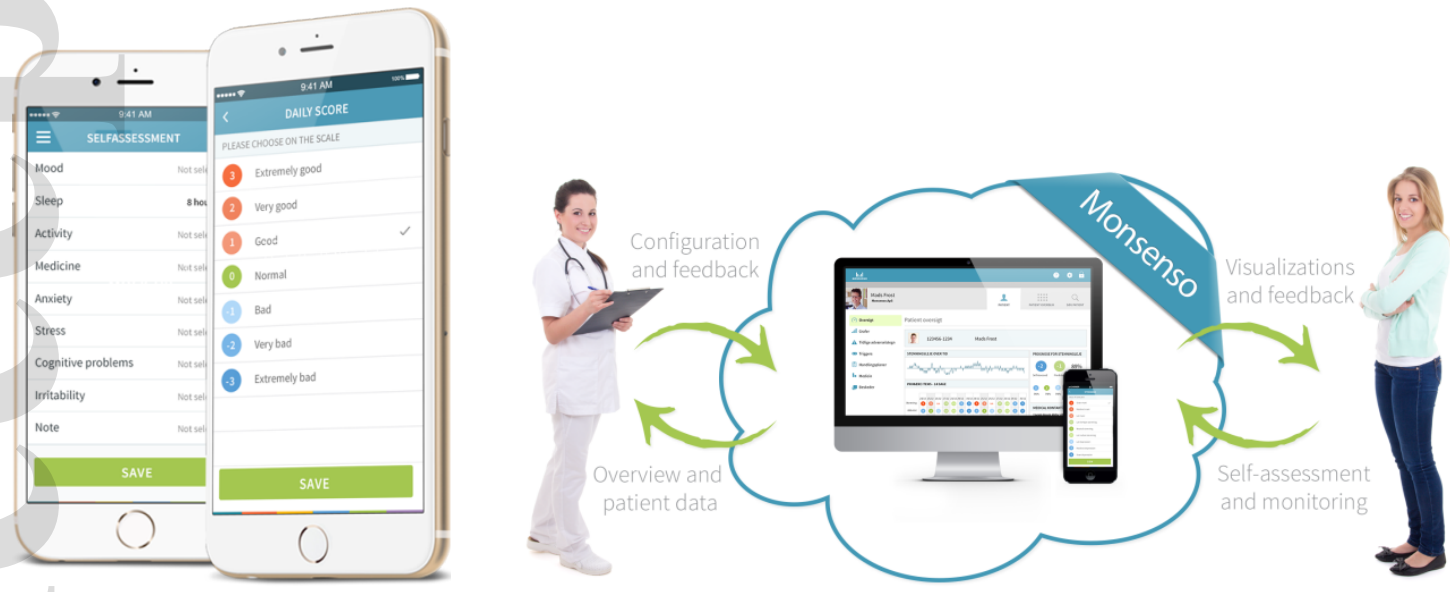

Figure 2. The Monsenso system for smartphones 


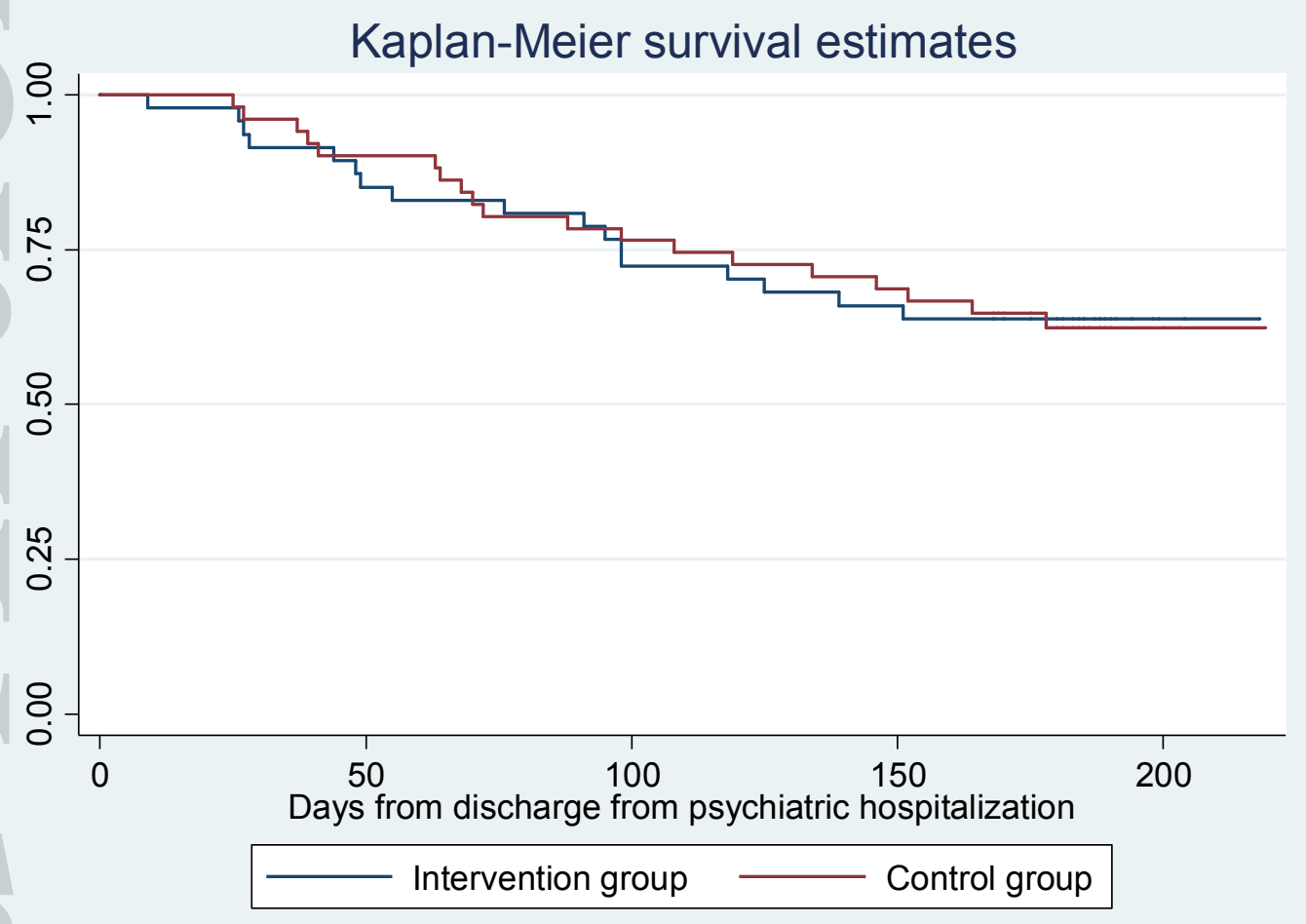

Figure 3. Kaplan-Meier curve of the ITT analysis on time to psychiatric readmission 
Table 1. Socio-demographic and clinical characteristics of study participants in the RADMIS trial at baseline, N=98

\begin{tabular}{|c|c|c|}
\hline Randomization group & Intervention group & Control group \\
\hline $\mathrm{N}$ & 47 & 51 \\
\hline \multicolumn{3}{|l|}{ Socio-demographic data } \\
\hline Age, years & $41.6(13.3)$ & $43.7(13.7)$ \\
\hline Female sex, \% (n) & $51.7(24)$ & $48.3(27)$ \\
\hline In relationship, \% (n) & $36.2(17)$ & $40.8(21)$ \\
\hline Education after primary school, years & $5.4(3.03)$ & $5.1(3.2)$ \\
\hline Full-time employed, \% (n) & $14.9(7)$ & $6.4(3)$ \\
\hline Part-time employed, \% (n) & $10.6(5)$ & $6.4(3)$ \\
\hline Student, \% (n) & $12.8(6)$ & $14.9(8)$ \\
\hline \multicolumn{3}{|l|}{ Clinical history } \\
\hline Bipolar disorder type I diagnosis, \% (n) & $59.6(28)$ & $56.9(29)$ \\
\hline Prior psychiatric admissions, number & $3[2-8]$ & $3[2-8]$ \\
\hline Prior depressive episodes, number & $6[4-10]$ & $5[2-10]$ \\
\hline Prior manic episodes, number & $5[2.5-10]$ & $5[2-11]$ \\
\hline Prior suicidal attempt, \% (n) & $54.4(26)$ & $36.7(19)$ \\
\hline Prior ECT, \% (n) & $27.3(13)$ & $31.2(16)$ \\
\hline First degree relative with psychiatric disorder, \% (n) & $75.0(35)$ & $59.2(30)$ \\
\hline HDRS-17", Baseline & $6[2-12]$ & $10[6-16]$ \\
\hline HDRS-17, 6 months & 7 [3-13] & $8[4-10]$ \\
\hline YMRS $^{* *}$, Baseline & $3[0-7]$ & $2[0-6]$ \\
\hline YMRS, 6 months & $0[0-2]$ & $0[0-6]$ \\
\hline FAST $^{* * *}$, Baseline & $33.07(11.19)$ & $34.5(11.82)$ \\
\hline FAST, 6 months & $21.9(13.49)$ & $24.9(14.77)$ \\
\hline
\end{tabular}

Data are mean (SD), median [IQR] or \%(n) unless otherwise stated; ${ }^{*}$ Hamilton Depression Rating Scale 17-items;

${ }^{* *}$ Young Mania Rating Scale; ${ }^{* * *}$ Functional Assessment Short Test 
Table 2. Estimated differences in primary and secondary outcomes between the intervention group and the control group (control group serve as reference), $\mathrm{N}=98$

\begin{tabular}{|c|c|c|c|c|c|c|c|c|c|}
\hline & \multicolumn{3}{|c|}{ Model $1^{\mathrm{a}}$} & \multicolumn{3}{|c|}{ Model $2^{b}$} & \multicolumn{3}{|c|}{ Model $3^{c}$} \\
\hline & Hazard Rate & $95 \% \mathrm{Cl}$ & $p$ & Hazard Rate & $95 \% \mathrm{Cl}$ & $p$ & - & - & - \\
\hline Psychiatric readmission & 1.00 & $0.52 ; 1.93$ & 0.99 & 1.05 & $0.52 ; 1.85$ & 0.88 & & & \\
\hline Depressive episodes $^{\mathrm{d}}, \mathrm{n}=57$ & 0.65 & $0.19 ; 2.13$ & 0.48 & 0.55 & $0.16 ; 1.89$ & 0.34 & - & - & - \\
\hline Manic episodes ${ }^{e}, n=93$ & 0.89 & $0.12 ; 6.25$ & 0.91 & 0.87 & $0.12 ; 6.25$ & 0.89 & - & - & - \\
\hline Affective episodes regardless polarity, $n=57$ & 0.64 & $0.30 ; 1.41$ & 0.28 & 0.63 & $0.29 ; 1.37$ & 0.24 & & & \\
\hline & $\begin{array}{c}\text { Difference } \\
\text { between } \\
\text { groups }\end{array}$ & $95 \% \mathrm{Cl}$ & $p$ & $\begin{array}{c}\text { Difference } \\
\text { between groups }\end{array}$ & $95 \% \mathrm{Cl}$ & $\mathrm{p}$ & $\begin{array}{c}\text { Difference } \\
\text { between groups }\end{array}$ & $95 \% \mathrm{Cl}$ & $p$ \\
\hline Duration of psychiatric readmission (days) & 3.20 & $-5.25 ; 11.65$ & 0.46 & 3.67 & $-4.77 ; 12.11$ & 0.39 & - & - & - \\
\hline HDRS-17* & -0.20 & $-2.55 ; 2.15$ & 0.87 & -0.11 & $-2.50 ; 2.29$ & 0.93 & - & - & - \\
\hline Sub-analyses on HDRS 17-items & & & & & & & & & \\
\hline 1A. HDRS-17 items baseline $\geq 7, n=34$ & 2.53 & $0.071 ; 4.99$ & 0.044 & 2.55 & $0.08 ; 5.02$ & 0.050 & - & - & - \\
\hline 1B. HDRS-17 items baseline $<7, n=64$ & -0.38 & $-1.77 ; 1.02$ & 0.60 & -0.39 & $-1.73 ; 0.95$ & 0.59 & - & - & - \\
\hline HDRS-6 $6^{n+1}$ & -1.49 & $-3.32 ; 0.34$ & 0.11 & -0.88 & $-2.80 ; 1.04$ & 0.37 & - & - & - \\
\hline YMRS $^{* *}$ & 1.93 & $0.15 ; 3.31$ & 0.034 & 1.89 & $0.0078 ; 3.78$ & 0.050 & - & - & - \\
\hline Sub-analyses on YMRS & & & & & & & & & \\
\hline 2A. YMRS baseline $\geq 7, n=20$ & 4.17 & $1.07 ; 7.27$ & 0.008 & 4.17 & $1.07 ; 7.27$ & 0.008 & - & - & - \\
\hline 2B. YMRS baseline $<7, n=78$ & 0.048 & $-0.87 ; 0.97$ & 0.92 & 0.048 & $-0.87 ; 0.97$ & 0.92 & - & - & - \\
\hline FAST $^{* * * *}$ & -1.78 & $-6.14 ; 2.59$ & 0.43 & -3.03 & $-7.43 ; 1.37$ & 0.18 & -1.91 & $-5.41 ; 1.59$ & 0.29 \\
\hline
\end{tabular}

${ }^{a}$ Adjusted for outcome variable at baseline; ${ }^{b}$ Adjusted for outcome variable at baseline, age and gender; ${ }^{\mathrm{c}}$ Adjusted for outcome variable at baseline, age and gender and HDRS-17 and YMRS scores; ${ }^{\mathrm{d}}$ Defined as HDRS-17 $\geq 13 ;{ }^{\text {e }}$ Defined as YMRS $\geq 13$; ${ }^{*}$ The Hamilton Depression Rating Scale 17 -items; ${ }^{* *}$ The Young Mania Rating Scale; ${ }^{* * *}$ The Hamilton Depression Rating Scale 6-items; ${ }^{* * * *}$ The Functional Assessment Short Test 
Table 3. Estimated differences in tertiary outcomes between the intervention group and the control group (control group serve as reference), $\mathrm{N}=98$

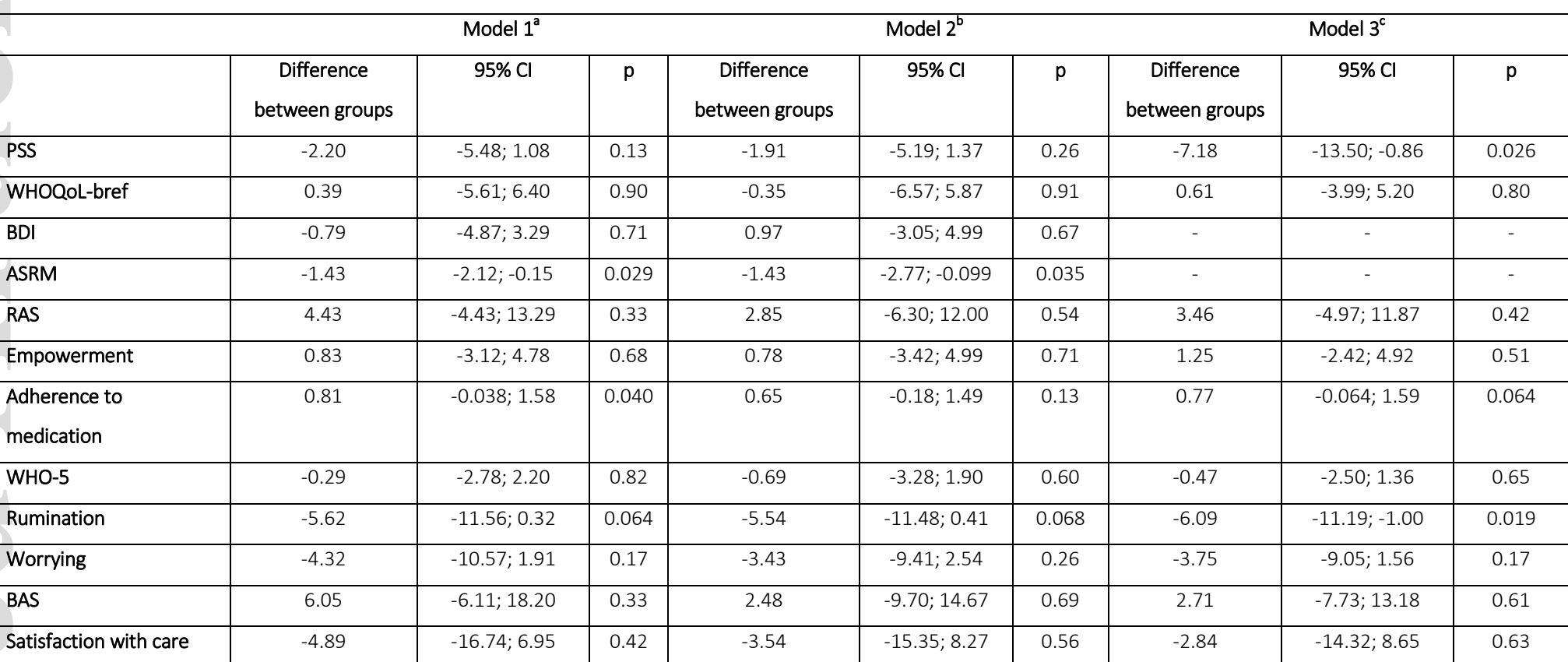

${ }^{a}$ Adjusted for outcome variable at baseline; ${ }^{b}$ Adjusted for outcome variable at baseline, age and gender; ${ }^{c}$ Adjusted for outcome variable at baseline, age and gender and $\mathrm{HDRS}-17$ and YMRS scores

PSS: Cohen's Percieved Stress Scale; WHOQoL-bref: World Health Organization Quality of Life - short version; BDI: Becks Depressive Inventory; ASRM: The Altman Self Rating scale for Mania; RAS: The Recovery Assessment Scale; Empowerment: Roger's Empowerment Scale; Adherence to medication: The Medicine Adherence Rating Scale; WHO-5:

The WHO Well-being Index; Rumination: The Ruminative Response Scale; Worrying: The Penn State Worry Questionnaire; BAS: Behavioral Activation Scale; Satisfaction with care:

The Verona Satisfaction Scale-Affective Disorder

This article is protected by copyright. All rights reserved 
Figure 1

The RADMIS Trial - Flow Diagram

Patients with a bipolar disorder diagnosis discharged from psychiatric hospitalization within the Mental Health Services, Capital Region of Denmark during the period from May 2017 to August 2019

Eligible but excluded due to ( $n=278$ ):

- Didn't want to participate in a research study $(n=110)$

- Didn't want smartphone-based monitoring ( $n=48$ )

- Not possible to get in contact with the patient $(n=89)$

- Other reasons not specified $(n=31)$
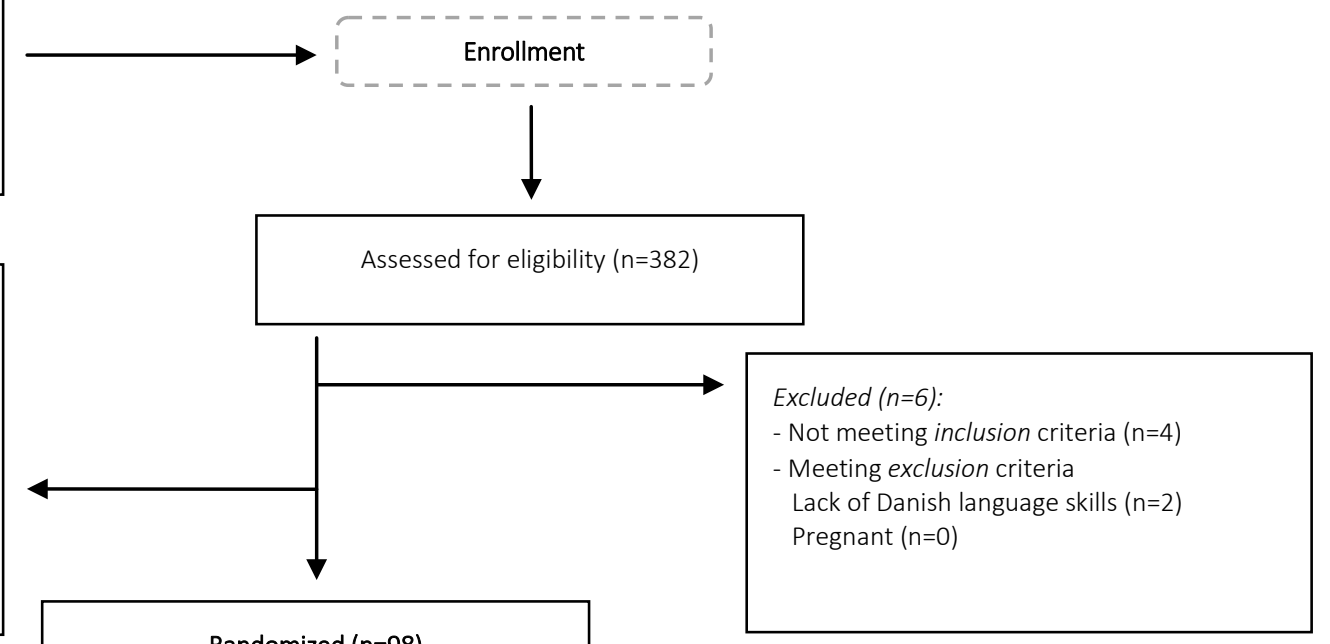

Randomized ( $\mathrm{n}=98$ )

Allocated to intervention group:

The Monsenso system $(n=47)$



- Withdraw from study $(n=3)$

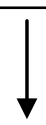

- Completed assessments ( $n=37)$

- Withdraw from study $(n=3)$

Analysed ( $n=47)$

- Excluded from analysis $(n=0)$

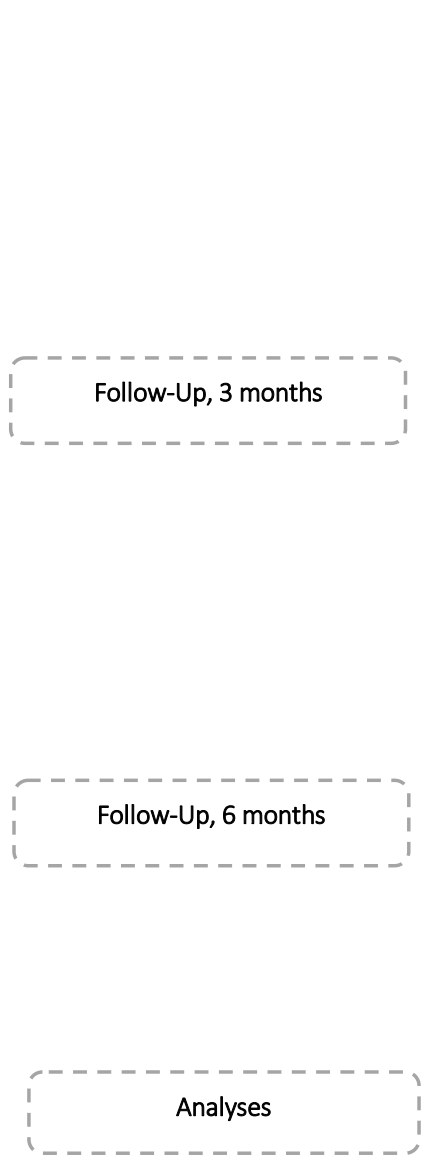

Allocated to control group: Use of smartphone for normal communicative purposes $(n=51)$

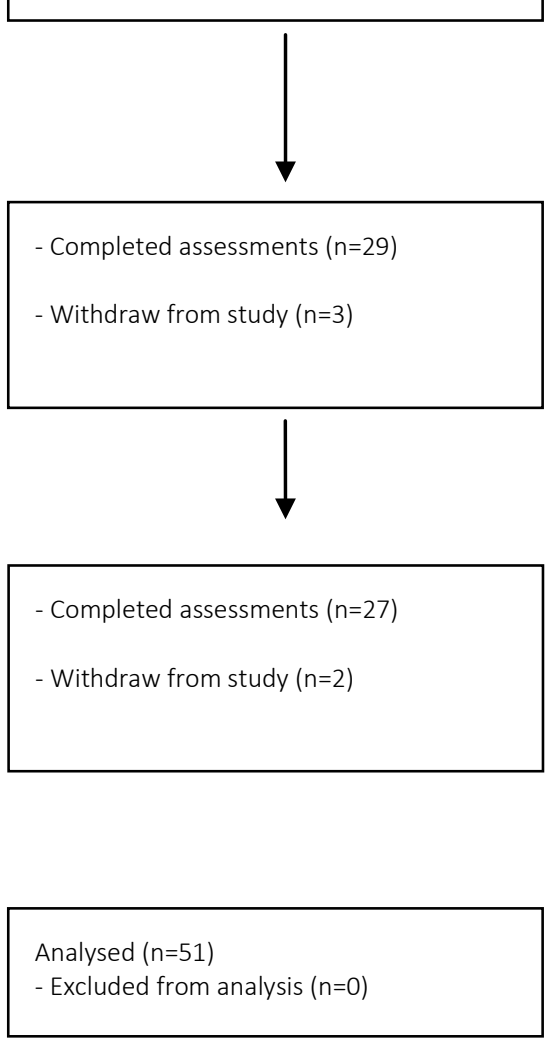

